\title{
ASSESSMENT OF THREE PHARMACOLOGICAL AGENTS FOLLOWING ARTHROCENTESIS FOR THE TREATMENT OF INTERNAL DERANGEMENT OF THE TEMPOROMANDIBULAR JOINT; A CLINICAL STUDY
}

\author{
Dalia A. Radwan*, Hesham El- Hawary*, Mohamed A. Eissa ** and Moataz Bahaa El- Din ${ }^{* * *}$
}

\begin{abstract}
Temporomandibular disorder (TMD) includes disorders of the temporomandibular joint (TMJ) and masticatory muscles and their associated structures. It is characterized by pain, joint sounds and restricted mandibular movement. Pharmacological agents commonly used for the treatment of TMDs include non-steroidal anti-inflammatory drugs (NSAIDs), opioids, corticosteroids, muscle relaxants, antidepressants, anticonvulsants and benzodiazepines.

Objectives: In the present study, we compared the clinical outcome of intra-articular injections of NSAIDs, corticosteroids, Hyaluronic acid given after temporomandibular joint arthrocentesis.

Materials and methods: Twenty-seven patients suffering internal derangement of the temporomandibular joint and not responding to conservative therapy were randomly classified into three equal groups. In group A joint lavage was followed by $1 \mathrm{ml}$ of piroxicam injection. In group B joint lavage followed by $1 \mathrm{ml}$ of dexamethasone injection and in group $\mathrm{C}$ joint lavage was followed by $1 \mathrm{ml}$ of Hyalgan injection. The treatment outcome was evaluated clinically by measuring the maximal mouth opening in mm at 2 weeks and 3 months postoperatively. Pain was measured using the Visual Analogue Scale at the study intervals. These data were statistically analyzed.
\end{abstract}

Results: All drugs were able to demonstrate a reduction in pain intensity and improvement in mouth opening at 2 weeks and 3 months postoperatively. No significant differences in treatment success were found among the three groups $(\mathrm{P}>0.05)$ through the study intervals.

Conclusion: We concluded that arthrocentesis with Piroxicam, dexamethasone or Hyalgan are similarly effective and are promising methods in relieving the symptoms of TMJ with nonreducing disc displacement. Additional prospective studies are required to confirm the adequate dosage of each treatment protocol, frequency of the injections required and combination between those protocols and other modalities in order to achieve long-term results.

KEYWORDS: Arthrocentesis, internal derangement, piroxicam, dexamethasone, hyaluronic acid.

\footnotetext{
* Associate Professor of Oral and Maxillofacial Surgery, Cairo University.

** Lecturer of Oral and Maxillofacial Surgery, Cairo University

*** Assistant Lecturer of Oral and Maxillofacial Surgery, British University in Egypt.
} 


\section{INTRODUCTION}

Internal derangement (ID) of the temporomandibular joint is a local malalignment of the joint components, which affects its smooth movement. ${ }^{1}$ ID is categorized into disc displacement with or without reduction. ${ }^{2}$ More precise classifications were offered by Wilkes ${ }^{3}$ Demitroulis ${ }^{4}$ and recently by Roman, el al ${ }^{5}$ based on MRI findings. These disorders may cause pain, clicking or crepitus sounds, limitation of jaw opening and deviation in jaw functions. ${ }^{6}$

Etiology of TMJ ID is still considered of a multifactorial nature where trauma, psychological stress, malocclusion, bruxism together with inflammation are the most accused of causing ID. ${ }^{7-9}$ Arthroscopic studies have clarified the role of inflammation of synovium, capsule or retrodiscal tissues in causing TMJ related pain. ${ }^{10-14}$

Because of insufficient knowledge of the etiology of ID, the treatment is empirical and usually addresses symptoms especially pain which is the most common chief complaint. A wide variety of treatment modalities have been introduced in management of TMJ ID starting with simple, conservative approaches ${ }^{6}$ to arthroscopy either simple lysis and lavage ending with a more complicated arthroscopic techniques as anterior disc and capsular release. ${ }^{15}$

Arthrocentesis is a treatment modality that breaks down joint adhesions and removes inflammatory mediators from the superior joint space, rather than repositioning the disc. ${ }^{16}$ It increases the range of motion and function as well as reduces pain. ${ }^{17}$ It also proved to be a minimally invasive, relatively safe and can be done on outpatients under local anesthesia. ${ }^{18}$

To enhance the outcome of arthrocentesis, postoperative intra-articular injection of various substances as Local anesthetics ${ }^{19,20}$, morphine ${ }^{19}$, corticosteroids ${ }^{21,22}$, sodium hyaluronate ${ }^{23}$, tenoxicam, celecoxib ${ }^{24}$ and piroxicam ${ }^{25}$ were used, to benefit from their analgesic, anti-inflammatory or lubricator properties.
Piroxicam is a non-steroidal anti-inflammatory drug. It is used to relief post-operative pain and gives an analgesic effect. ${ }^{26}$ The parenteral formulation of piroxicam has an aqueous base, without an organic stabilizer and since solvent for injection is distilled water, this formulation offers the potential for intra-articular administration. ${ }^{27}$ Some authors have reported intra-articular corticosteroid injections to reduce pain and improve function. ${ }^{28}$ Glucocorticoids are yet the most effective anti-inflammatory drugs available, promoting symptomatic improvements of a series of clinical manifestations. It was found that corticosteroids have a potent anti-inflammatory effect on synovial tissues and are known to reduce effusion, decrease pain, and bring about an increase in the range of motion. ${ }^{29}$

On the other hand, Hyaluronic acid (HA) has also been shown to reduce friction and pain and improve joint mobility by restoring soft-tissue lubrication and repair articular cartilage nutrition. ${ }^{30}$ It has been suggested that exogenous administration of hyaluronate stimulates hyaluronate production by synoviocytes inside the joint, reducing friction and thus protecting articular structures. ${ }^{31}$

Therefore, the aim of this study was to compare the clinical outcome of intrarticular injections of piroxicam, dexamethasone and hyaluronic acid following arthrocentesis in patients with nonreducing disc displacement.

\section{Methodology}

This study was conducted on patients selected from those attending the outpatient clinic in Oral and Maxillofacial Surgery Department, Faculty of Dentistry, Cairo University. Cairo University Review Board and Ethics Committee approved the study.

The inclusion criteria was to fulfill at least two of the following diagnostic criteria for internal derangement of TMJ as suggested by the American Association of Oral and Maxillofacial Surgery 
(AAOMS). ${ }^{32}$ These included pain in the region of TMJ, popping, clicking sounds during condylar movement, Limitation of mandibular movement not exceeding $25 \mathrm{~mm}$ (i.e. condylar rotation with no translation), Clinical, and radiographic evidence of organic changes in the integrity of the joint.

The selected patients were healthy adults who were free from any systemic diseases that might interfere with TMJ treatment. MRI was done for the patients and they were subjected to conservative therapy for 4 weeks in terms of soft diet, splint therapy, medical treatment and physiotherapy to exclude the responders and to keep only those who were noncompliant to the previous treatment modalities.

Twenty-seven patients who had anterior disc displacement without reduction and did not respond to the conservative therapy were included in the study. Their ages ranged from 20 to 43 years and they approved to participate in the study through signed informed consents.

\section{Arthrocentesis technique}

Under aseptic conditions, auriculotemporal nerve block anesthesia (Fig. 1) was induced using one carpule of Mepivacaine (mepivacaine $\mathrm{HCl}$

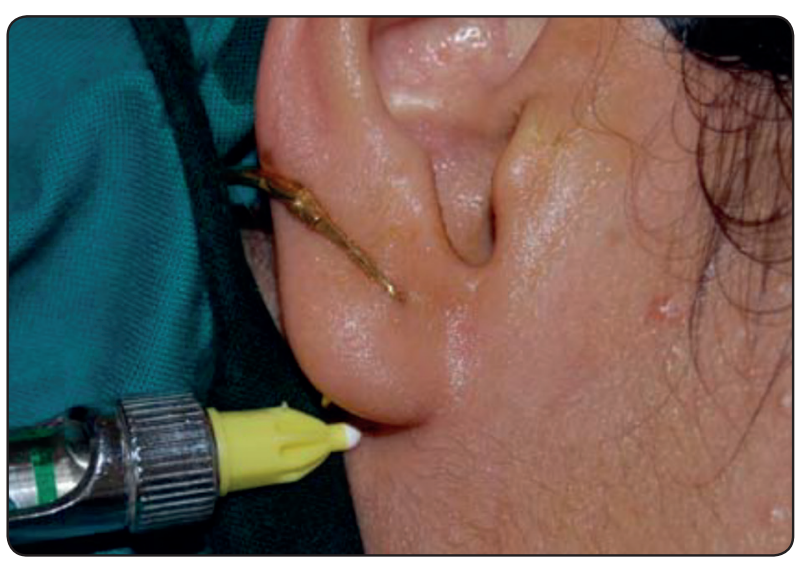

Fig. (1) Auriculotemporal nerve block anesthesia.
2\% with levonordefrin 1: 20 000, Alexandria Co. pharmaceuticals, Alexandria, Egypt). External auditory canal is protected from accumulation of blood and fluid by a cotton pellet. The surgical technique of Nitzan et al. ${ }^{33}$ was followed. The first needle (inlet), corresponding to the glenoid fossa, was marked $10 \mathrm{~mm}$ from the mid-tragus and 2 $\mathrm{mm}$ below the canthotragal line. A second needle (outlet), corresponding to the articular eminence, was marked $10 \mathrm{~mm}$ from the first point and 10 $\mathrm{mm}$ below the line. A total amount of $200 \mathrm{ml}$ of normal saline solution was introduced into the inlet needle (Fig 2). The joint is manipulated through opening, closing, protrusive and lateral excursions of the mandible to establish free flow of the solution and release adhesions, at the end of procedure the outflow needle was removed and first needle was used to inject the different medications. Patients were randomly divided into three equal groups.

Group A: 1ml Feldene (1 ml ampoule Piroxicam manufactured by Pfizer S.A.E Cairo, Egypt) was injected into the superior joint space at the end of the lavage.

Group B: 1ml dexamethasone (produced by Amriya for pharmaceutical industries, Alex, Egypt) was injected into the superior joint space at the end of the lavage.

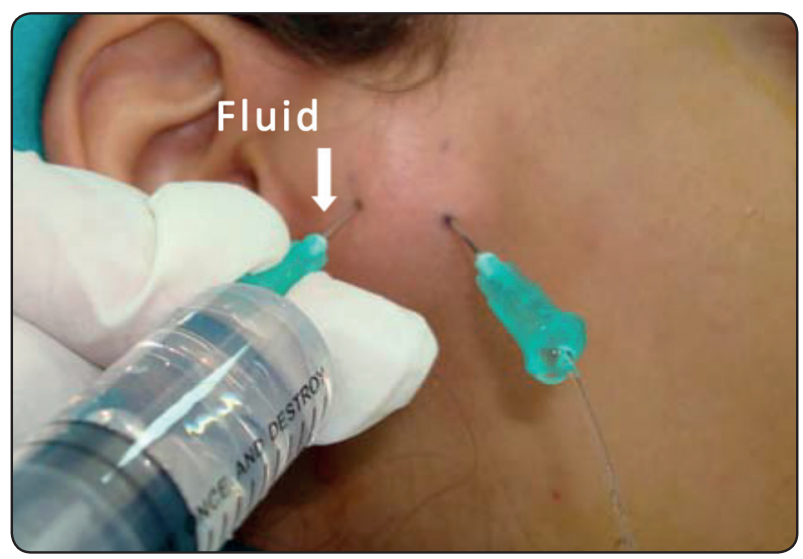

Fig. (2) Establishment of free flow of the washing solution. 
Group C: 1ml Hyalgan (Hyaluronic acid sodium salt, Manufactured by Fidia Farmaceutici S.P.A, Italy) was injected into the superior joint space at the end of the lavage.

Finally, the inlet cannula was withdrawn, and the preauricular area was covered with a sterile pressure dressing for the next 24 hours. Patients were instructed to apply ice packs over the area of injection to reduce the postoperative pain and edema then shift to warm application the day after the procedure 5 times a day for 2 weeks. Emox $500 \mathrm{mg}$ tablets (Amoxycillin (as trihydrate) $500 \mathrm{mg}$ manufactured by Egyptian Int Pharmaceutical Industries Co. E.I.P.I.C.O, Egypt) was prescribed as antibiotic for 1 week. Cataflam $50 \mathrm{mg}$ tablets (Novartis Parma. SAE, Cairo C.C.R-111108 under license from Novartis Pharma AG, Basle, Swizerland) was prescribed as an analgesic and antiinflammatory for 1 week. Patients were instructed to maintain soft diet as tolerated by the patient for the following 10 days with gradual transformation to normal diet within 2 weeks.

Follow-up was done at 2 weeks and 3 months post operatively Patients were evaluated for pain upon mouth opening, which was recorded according to the pain Visual Analog Scale (VAS) on a range of 0-10 with the extremes being 'no pain' and 'pain as bad as the patient ever experienced. Moreover, jaw range of motion function in millimeters was assessed in terms of maximum interincisal opening (MIO) measured by the maximum interincisal distance (Fig 3).

Data was presented as mean \pm SD. Analysis of variance test was used for comparison between groups. The significance level was set at $\mathrm{P}$ value less than or equal to 0.05 . Statistical analysis was performed with IBM SPSS Statistics, version 20 (SPSS, Inc., an IBM Company, IBM Corporation, NY, USA.), for Windows.

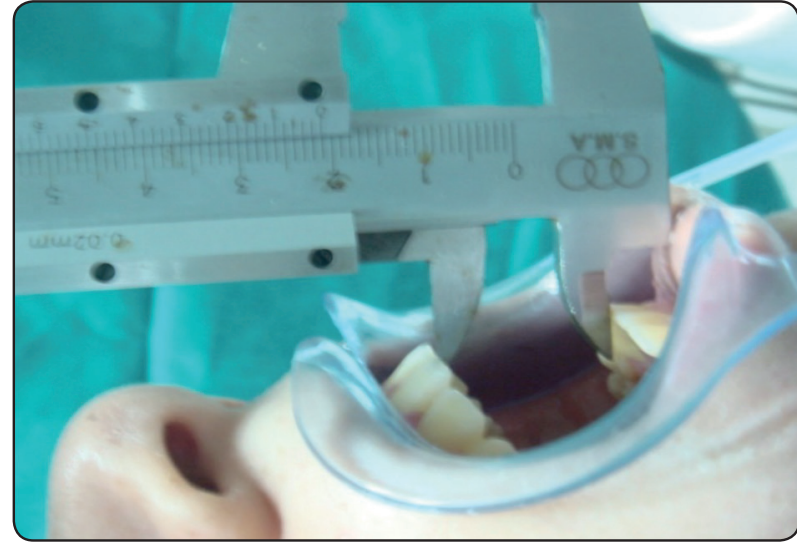

Fig. (3) Measurement of maximum interincisal opening.

\section{RESULTS}

A total of twenty seven patients with painful TMJ and limited opening secondary to disc displacement without reduction were enrolled in the present study. The patients' age ranged between 20 to 43 years old with a mean of 31 years old. They were randomly divided into three equal groups: group A for Piroxicam injection, group B for dexamethasone injection and group $\mathrm{C}$ for Hyalgan, following saline lavage. No adverse effects related to injected materials or arthrocentesis were observed during treatment and follow-up periods except for two patients who had extravasation of fluid into surrounding tissue, which eventually resolved in two days.

In terms of pain intensity, there was a statistically significant reduction $(\mathrm{P}<0.05)$ in VAS measurements in each group at 2 weeks and 3 months postoperatively in comparison with their corresponding preoperative measurements (Table 1), yet the VAS scores did not vary significantly among the three groups at 2 weeks and 3 months postoperatively ( $\mathrm{P}>0.05)$, (Fig. 4).

In terms of MIO, a limited mouth opening was obvious preoperatively, and it improved postoperatively. there was a statistically significant increase in maximal mouth opening $(\mathrm{P}<0.05)$ in 
each group at 2 weeks and 3 month postoperatively, in comparison with their corresponding preoperative measurements (Table 2), yet the mean MIO scores did not vary significantly among the three groups at 2 weeks and 3 months postoperatively $(\mathrm{P}>0.05)$ (Fig. 5).

TABLE (1) Comparison of pre and post-treatment mean VAS values

\begin{tabular}{|l|c|c|c|}
\hline & $\begin{array}{c}\text { Group A } \\
\text { mean } \pm \text { SD }\end{array}$ & $\begin{array}{c}\text { Group B } \\
\text { mean } \pm \text { SD }\end{array}$ & $\begin{array}{c}\text { Group C } \\
\text { mean } \pm \text { SD }\end{array}$ \\
\hline Preoperative & $7.33 \pm 1.4$ & $9.67 \pm 0.81$ & $10.00 \pm 0$ \\
\hline 2 weeks & $4.89 \pm 1.8$ & $6.17 \pm 3.92$ & $7.50 \pm 2.51$ \\
\hline 3 months & $2.33 \pm 1.9$ & $3.67 \pm 3.88$ & $2.83 \pm 3.71$ \\
\hline P-value & \multicolumn{3}{|c|}{$<0.05$} \\
\hline
\end{tabular}

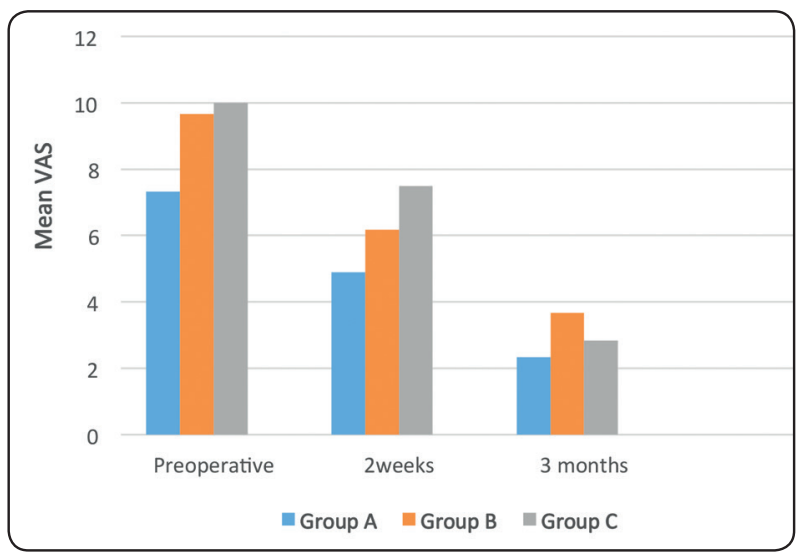

Fig. (4) Bar chart representing the changes by time in mean VAS of the three groups.

TABLE (2) Comparison of pre and post-treatment mean MIO values

\begin{tabular}{|l|c|c|c|}
\hline & $\begin{array}{c}\text { Group A } \\
\text { mean } \pm \text { SD }\end{array}$ & $\begin{array}{c}\text { Group B } \\
\text { mean } \pm \text { SD }\end{array}$ & $\begin{array}{c}\text { Group C } \\
\text { mean } \pm \text { SD }\end{array}$ \\
\hline Preoperative & $18.8 \pm 4.0$ & $26.67 \pm 3.9$ & $25.10 \pm 2.2$ \\
\hline 2 weeks & $31.8 \pm 4.9$ & $34.33 \pm 8.2$ & $33.00 \pm 5.5$ \\
\hline 3 months & $33.9 \pm 6.1$ & $36.33 \pm 8.9$ & $37.83 \pm 8.0$ \\
\hline P-value & \multicolumn{3}{|c}{$<0.05$} \\
\hline
\end{tabular}

SD: Standard deviation

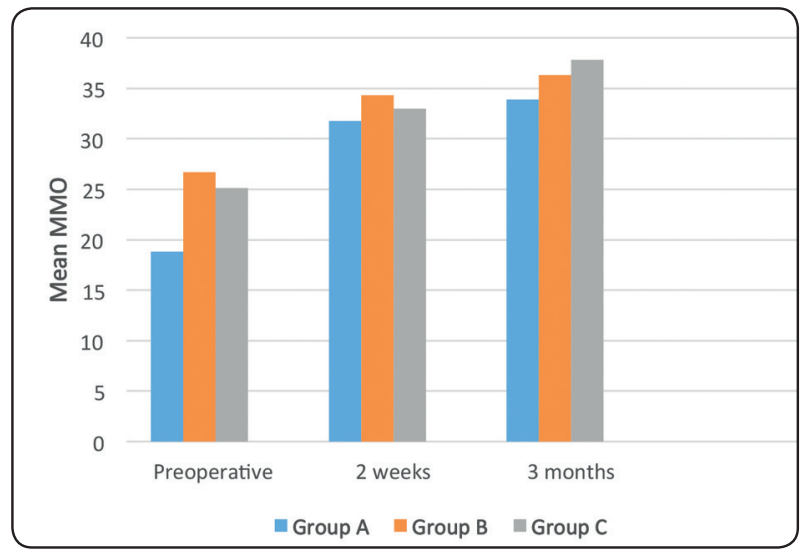

Fig. (5) Bar chart representing changes by time in mean maximum interincisal opening of the three groups

\section{DISCUSSION}

TMD it is a collective term that includes disorders of the temporomandibular joint and the masticatory muscles. TMDs are characterized by pain, joint sounds and restricted mandibular movement. ${ }^{34}$ These symptoms are interrelated, as increased pain causes decreased maximal opening. ${ }^{35},{ }^{36}$

The Temporomandibular joint is a highly adaptive organ that constantly adjusts to the functional demands made on it by means of remodeling. ${ }^{37}$. Arthrocentesis results in elimination of the vacuum in the superior joint space and facilitation of disc and condyle translation. ${ }^{38}$ Even if the disc is not reduced, an increase in $\mathrm{MIO}$ and decrease in pain are established. ${ }^{39}$

The present investigation was designed to obtain a deeper understanding of the effectiveness of TMJ arthrocentesis under three different treatment protocols in a comparative clinical trial in terms of reduction of pain intensity and increase in the interincisal mouth opening. The procedure was performed under local anesthesia, lavage was done by sterile normal saline followed by injection of piroxicam (group A), dexamethasone (group B) and HA (group C).

Some postoperative complication were recorded in this study, two patients had pre-auricular swelling due to extra-articular fluid extravasation which resolved by the second postoperative day. 
The clinical results of group A revealed a significant reduction in pain intensity besides a significant increase in the measurements of maximal inter-incisal opening. These findings are in agreement with the results obtained by Al-said et al. ${ }^{38}$ The results were justified by Ethunandan and Wilson, ${ }^{40}$ who stated that, as Piroxicam is NSAIDs, subsequently it removes the inflammatory mediators, alters the intra-articular pressure and reduces synovial inflammation. Dionne and Berthold ${ }^{41}$ added that piroxicam concentrates in the synovium rather than in the cartilage.

The statistically significant improvement in pain and mouth opening in study group B is in agreement with the findings of Giradd et al. ${ }^{42}$ They elucidated that Corticosteroids have a potent anti-inflammatory effect on synovial tissue. They are also known to reduce effusion, decrease pain and bring about an increase in range of motion of synovial joints. ${ }^{42}$ Intra-articular corticosteroid injection provides, long-term palliative effects on subjective symptoms and clinical signs of TMJ pain. ${ }^{43,44}$ These results are also covenant with a systematic review showing that HA and glucocorticoids had the same short and long-term effects on improvement of clinical symptoms. ${ }^{45}$ On the other hand, the results disagree with that of Gencer et al, ${ }^{46}$ who found that HA is better in relieving pain when compared with corticosteroid injection in TMJ disorders.

Regarding group $\mathrm{C}$, the results revealed a significant decrease in TMJ pain following HA injection after arthrocentesis. Our results are in accordance with Morey-Mas et al, ${ }^{47}$ who attributed the reduction in pain intensity to the elimination of inflammatory mediators that causes pain. Gotoh et al added that injected sodium hyaluronate might show its analgesic effect through both, blocking pain receptors and HA's ability to penetrate synovial tissue to prevent further adhesions. ${ }^{48,49}$ Maximum mouth opening increased relatively at the end of the 3 months follow up. This improvement in jaw mobility might be due to analgesic, lubricator effects of hyaluronic acid. Hishashi et al, suggested that it minimizes wear and tear mechanically and plays a role in nutrition of the avascular parts of the disc and condylar cartilage. ${ }^{50}$

When comparing the outcomes of the three groups, the differences were statistically non-significant at the estimated time points of the postoperative period. This study clearly demonstrated that intra-articular injections of Piroxicam, corticosteroids or HA after saline lavage reduced pain and inflammation and improved mandibular function. Consequently, this validates the effectiveness of all methods in treatment of TMD patients. The success of treatment was to increase the range of motion and relieve the functional pain of the TMJ through effective lysis and lavage of the superior joint space rather than to reduce the displacement.

\section{CONCLUSION}

We concluded that arthrocentesis with Piroxicam, dexamethasone or Hyaluronic acid are similarly effective and are promising methods in relieving the symptoms of TMJ with non-reducing disc displacement. Additional prospective studies with longer-term evaluation are required to confirm the adequate dosage of each treatment protocol, frequency of the injections required and combination between those protocols and other modalities.

\section{REFERENCES}

1. Nyberg J, Adell R, Svensson B: Tempromandibular joint discectomy for treatment of unilateral internal derangement- a 5-year follow-up evaluation. Int J Oral Maxillofac Surg 2004; 33:8-12.

2. Miloro M: Peterson's principles of oral and maxillofacial surgery. Canada: BC Decker, 2004. Second edition.

3. Wilkes $\mathrm{CH}$ : Internal derangements of the temporomandibular joint. Pathological variations. ArchOtolarnyngol Head Neck Surg 1989: 115:469-477

4. Dimitroulis G: A new surgical classification for temporomandibular joint disorders. Int J Oral Maxillofac Surg 2013; 42: 218-222

5. Kowalchuk R, Kaplan K, Caplash J, Block P: Temporomandibular Joint Internal Derangement Score (TIDS): novel magnetic resonance imaging assessment score and its relation to invasive treatment in patients with clinical temporomandibular joint pathology, Heliyon 4 (2018) e00916. 
6. Brennan PA, Ilankovan V: Arthrocentesis for temporomandibular Joint pain dysfunction syndrome. J Oral Maxillofac Surg 2006; 64:949-951.

7. Ahmad M, Schiffman: Tempromandibular joint disorders and orofacial pain. Dent clin North Am 2016; 60: 105-124

8. Ghurye S, McMillan R: Pain-related temporomandibular disorder - current perspectives and evidence-based management. Dent Update 2015; 42:533-536, 539-42, 545-6.

9. Roldán-Barraza C, Janko S, Villanueva J, Araya I, Lauer HC: A systematic review and meta-analysis of usual treatment versus psychosocial interventions in the treatment of myofascial temporomandibular disorder pain. J Oral Facial Pain Headache 2014; 28:205-222

10. Kardel R, Ulfgren A K, Reinholt F P, Holmlund A: Inflammatory cell and cytokine patterns in patients with painful clicking and osteoarthritis in the temporomandibular joint. Int J Oral Maxillofac Surg 2003; 32:390-396.

11. Seki H, Fukuda M, Iino M, Takahashi T, YoshiokaN: Immunohistochemical localization of cyclooxygenase-1 and -2 in synovial tissues from patients with internal derangement or osteoarthritis of the temporomandibular joint. Int $\mathbf{J}$ Oral Maxillofac Surg 2004; 33:687- 692.

12. Quinn JH, Kent JN, A, Lukiw WJ: Cyclooxygenase-2: Noise in the synovial tissue and fluid of dysfunctional temporomandibular joints with internal derangement. J Oral Maxillofac Surg 2000; 58:1229-1232.

13. Quinn JH, Bazan NG: Identification of prostaglandin E2 and leukotriene B2 in the synovial fluid of painful dysfunctional temporomandibular joints. J Oral Maxilofac Surg 1990; 48:968-971

14. Ogasawara T, Kitagawa Y, Ogawa T, Yamada T, Kawamura Y, Sano K: Inflammatory change in upper joint space in the temporomandibular joint with internal derangement on Gadolinium- enhanced MR imaging. Int J Oral Maxillofac Surg 2002; 31:252-256.

15. Miyamoto H, Sakashita H, Miyata M: Arthroscopic surgery of the temporomandibular Joint: Comparison of Two Successful Techniques. Br J Oral Maxillofac Surg 1999; 37:397.

16. Alpaslan C, Dolwick MF, Heft MW: Five-year retrospective evaluation of temporomandibular joint arthrocentesis. Int J Oral Maxillofac Surg. 2003; 32:263-267.

17. Günay Yapici Yavuz, Aydin Keskinruzgar. Galore. Evaluation of Complications of Arthrocentesis in the Management of the Temporomandibular Joint Disorders. International Journal of Health Sciences and Research 50 Vol.3; Issue: 2; April-June 2018
18. Shailesh Kumar, Kamini Kiran, Anurag Yadav: Temporomandibular joint arthrocentesis: A prospective study and audit of 500 joints of central india. J Int Soc Prevent Communit Dent 2018;8:124-9.

19. Zuniga JR, Ibanez C, Kozacko M: The analgesic efficacy and safety of intra-articular morphine and mepivicaine following temporomandibular joint arthroplasty. Int Journal Oral and Maxillofacial Surgery. 2007 36:922-927

20. Scott S, Neil R, Connelly R: Postoperative analgesia for outpatient arthroscopic knee surgery with inrta-articular bupivacaine and ketorolac. Anaesth Analg 1995; 80: 1154-7.

21. Fouda A: Association between Intra-Articular Corticosteroid Injection and Temporomandibular Joint Structure Changes. Int Arch Oral Maxillofac Surg. 2018; 2:015 Volume 2 I Issue 1

22. Davoudi A, Khaki, H, Mohammadi E, Daneshmand M, Tamizifar A, Bigdelou M, Ansaripoor F: Is arthrocentesis of temporomandibular joint with corticosteroids beneficial? A systematic review. Med Oral Pathol Oral Cir Bucal. 2018 May 1; 23 (3):e367-75

23. Effectiveness of Sequential Viscosupplementation in Temporomandibular Joint Internal Derangements and Symptomatology: A Case Series. Pain Research and Management Volume 2018, Article ID 5392538,

24. Ta LE, Dionne RA: Treatment of painful temporomandibular joints with a cyclooxygenase-2 inhibitor: A randomized placebo-controlled comparison of Celecoxib and Naproxen. Pain 2004 111(1-2):13-21.

25. Izdes S, Orhun S, Turanli S, Erkilic E, Kanbak O: The effects of preoperative inflammation on the analgesic efficacy of intraarticular piroxicam for outpatient knee arthroscopy. Anesth analg 2003; 97:1016-9.

26. Chan Woong Park, Kyung Wan Ma, Sun Woo Jang, Miwon Son and yung Joo Kang: Comparison of Piroxicam Pharmacokinetics and Anti-Inflammatory Effect in Rats after Intra-Articular and Intramuscular Administration. Biomol Ther ; 22(3), 260-266 (2014)

27. Dionne RA, Berthold CW. Therapeutic uses of nonsteroid anti-inflammatory drug in dentistry. Crit Rev Oral Biol Med 2001; 12: 315-30.

28. Giraddi GB, Siddaraju A, and Kumar B, Singh C. Internal derangement of temporomandibular joint: an evaluation of effect of corticosteroid injection compared with injection of sodium hyaluronate after arthrocentesis. J Oral Maxillofac Surg. 2012; 11:258-63.

29. Ghanem WA. Arthrocentesis and stabilizing splint are the treatment of choice for acute intermittent closed lock in 
patients with bruxism. J Craniomaxillofacial Surg. 2011; 39:256-60.

30. Li C, Long X, Deng M, Li J, Cai H, Meng Q. Osteoarthritic changes after superior and inferior joint space injection of hyaluronic acid for the treatment of temporomandibular joint osteoarthritis with anterior disc displacement without reduction: a cone-beam computed tomographic evaluation. J Oral Maxillofac Surg. 2015; 73:232-44.

31. Guarda-Nardini L, Stifano M, Brombin C, Salmaso L, Manfredini D. A one-year case series of arthrocentesis with hyaluronic acid injections for temporomandibular joint osteoarthritis. Oral Surg Oral Med Oral Pathol Oral Radiol Endod. 2007; 103:e14-22.

32. American Association of Oral and Maxillofacial Surgery. Criteria for TMJ meniscus surgery. Chicago, IL: American Association of Oral and Maxillofacial Surgery; 1984.

33. Nitzan DW, Dolwick MF, Martinez GA: Temporomandibular joint arthrocentesis: a simplified treatment for severe, limited mouth opening. J Oral Maxillofac Surg 1991; 49: 1163-1167.

34. Ouanounou, A, Goldberg M, Haas D A: Pharmacotherapy in Temporomandibular Disorders: A Review, J Can Dent Assoc 2017; 83:h7

35. Carvajal W, Laskin DM. Long-term evaluation of arthrocentesis for the treatment of internal derangements of the temporomandibular joint. J Oral Maxillofac Surg 2000; 58: 852-5.

36. Venancio RA, Camparis CM, Lizarelli RZ: Low intensity laser therapy in the treatment of temporomandibular disorders: a double-blind study. J Oral Rehabilitation 2005; 32: 800-7.

37. Pavan K, Haripriya C, Mohan AP, Brahmaji R: A Clinical Study of Efficacy of Hydrocortisone Compared With Hyaluronic Acid After Arthrocentesis in TMJ Disorders, Indian J Dent Adv 2016; 8(3): 141-147.

38. Al-said s n, shawky n, Ragab h r: comparative study of arthrocentesis with Or without using piroxicam in the Management of temporomandibular joint disorders, ADJ (2015) vol.40 pages:160-165

39. Al-Belasy FA, Dolwick MF. Arthrocentesis for the treatment of temporomandibular joint closed lock: a review article. Int J Oral Maxillofac Surg. 2007; 36:773-82.

40. Ethunandan M, Wilson AW. Temporomandibular joint arthrocentesis-more questions than answers. J Oral Maxillofac Surg 2006; 64: 952-5.
41. Dionne RA, Berthold CW. Therapeutic uses of nonsteroid anti-inflammatory drug in dentistry. Crit Rev Oral Biol Med 2001; 12: 315-30.

42. Giraddi GD, Siddaraju A, Kumar B, Singh C: Internal Derangement of Temporomandibular Joint: An Evaluation of Effect of Corticosteroid Injection Compared with Injection of Sodium Hyaluronate after Arthrocentesis. J. Maxillofac. Oral Surg 2012; 11(3):258-263.

43. Kopp S, Wenneberg B, Haraldson T, Carlsson GE: The Short-term Effect of Intra-articular Injections of Sodium Hyaluronate and Corticosteroid on Temporomandibular Joint Pain and Dysfunction. J Oral Maxillofac Surg 1985; 43(6):429-435.

44. Kopp S, Carlsson GE, Haraldson T, Wenneberg B: LongTerm Effect of Intra-Articular Injections of Sodium Hyaluronate and Corticosteroid on Temporomandibular Joint Arthritis. J Oral Maxillofac Surg 1987; 45(11):929- 935

45. Shi Z, Guo C, Awad M: Hyaluronate for temporomandibular joint disorders:systematic review. Cochrane Database Syst Rev 2003; 34:CD002970.

46. Gencer ZK, Oziriş M, Okur A, Korkmaz M, Saydam L: A comparative study on the impact of intra-articular injections of hyaluronic acid, tenoxicam and betametazon on the relief of temporomandibular joint disorder complaints. J Craniomaxillofac Surg 2014; 42:1117-1121.

47. Miguel-Angel Morey-Mas MA, Caubet-Biayna J, Sende LV, Iriarte-Ortabe JI: Sodium Hyaluronate Improves Outcomes After Arthroscopic Lysis and Lavage in Patients with Wilkes Stage III and IV Disease, J of Oral and Maxillofac Surgery Volume 68, Issue 5, May 2010, Pages 1069-1074.

48. Gotoh S, Miyazaki K, Onaya J, Sakamoto T, Tokuyasu K, Namiki O (1988) Experimental knee pain model in rats and analgesic effect of sodium hyaluronate (SPH). Folia Pharmacol Japon 92:17-27.

49. Gotoh S, Onaya J, Sakamoto T, Tokuyasu K (1988) Protective effect of sodium hyaluronate (SPH) against adhesion formation in flexor tendon of rats. Pharmacometrics 35:359-364

50. Hishashi N, Ishimaru JI, Kurita K et al (1997) The effect of hyaluronic acid on experimental temporomandibular joint osteoarthrosis in the sheep. J Oral Maxillofac Surg 55:1114 . 